\title{
Capsule endoscopic appearance of the small- intestinal mucosa in Whipple's disease and the changes that occur during antibiotic therapy
}

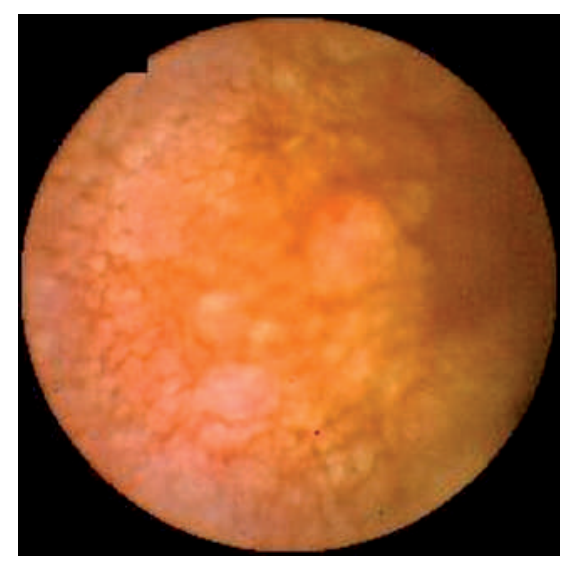

Figure 1 Pseudopolypoid structure of the mucosal surface in the proximal jejunum seen in the baseline capsule endoscopic examination in patient 1 .

To date there have been very few reports describing the macroscopic appearance of the small bowel in Whipple's disease as viewed by capsule endoscopy $[1,2]$. We report the capsule endoscopic findings in two patients with Whipple's disease before antibiotic therapy and during follow-up, using the M2A capsule endoscope (Given Imaging Ltd., Yoqneam, Israel).

Baseline capsule endoscopy in patient 1 showed massive villous atrophy in the mucosa of the duodenum and the proximal jejunum, a pseudopolypoid surface structure, and a whitish reticular pattern that was suggestive of dilated lymphatic vessels (Figure 1,2). Multiple erosions and villous denudation were also observed. The mucosa was affected continuously, around its whole circumference. These pathologic features were most pronounced in the duodenum and decreased in intensity as the capsule progressed distally. The distal jejunum and the ileum showed a discontinuous pattern of mucosal involvement, with only part of the circumference affected. Whitish spots on the surface of the mucosa were the most prominent finding in this part of the bowel (Figure 3). Follow-up capsule endoscopy in patient 1, 9 months after initiation of antibiotic treatment, showed findings in the duodenum that were unchanged

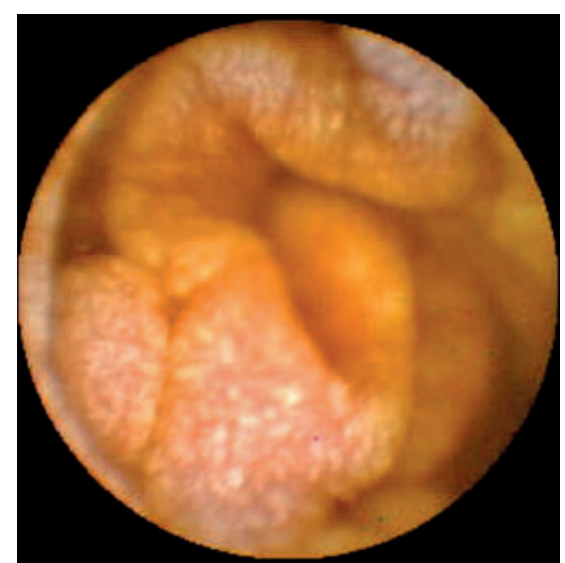

Figure 2 The mucosa of the proximal jejunum showed a whitish reticular pattern, suggestive of dilated lymphatic vessels (baseline capsule endoscopy in patient 1 ).

from the baseline appearance. In the proximal jejunum, the villous structure of the mucosa seemed intact; the surface was studded with whitish spots (Figure 4). In the distal jejunum and the ileum, the mucosa was macroscopically normal.

Hardly any villi or folds were visible in the duodenum and proximal jejunum in the baseline capsule endoscopy examination in patient 2. The surface of the mucosa had a whitish reticular pattern (Figure $\mathbf{5}$ ), and there were a very small number of erosions and areas of villous denudation (Figure 6). These features decreased in intensity as the capsule moved distally. In the distal ileum the only pathologic finding was whitish spots on the surface of the mucosa. In all parts of the small bowel the mucosa was affected continuously and around its whole circumference. At follow-up capsule endoscopy in patient 2, 29 months after the baseline examination (i.e. 17 months after termination of antibiotic therapy), the mucosa in all parts of the small intestine appeared normal.

We conclude that Whipple's disease does not always involve the entire small bowel in a continuous fashion, but that a discontinuous pattern of mucosal involvement can occur. Resolution of the mucosal

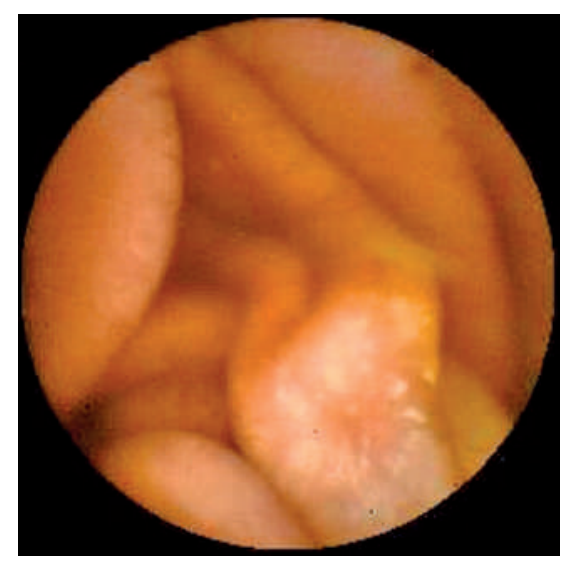

Figure 3 Baseline capsule endoscopy in patient 1 showed a discontinuous pattern of mucosal involvement in the distal ileum, with only part of the circumference affected. Whitish spots on the surface of the mucosa were the most prominent finding.

changes seems to start in the distal parts of the small bowel and progresses in a proximal direction, and antibiotic treatment can lead to complete normalization of the capsule endoscopic appearance of the small-intestinal mucosa in this condition.

\section{Acknowledgment}

Part of this work was made possible by the Whipple's Disease Project of the 5th Framework Program of the European Community (contract reference QLG1CT-2000-01049). Part of this paper was presented as an abstract at the 38th Annual Meeting of the Austrian Society of Gastroenterology and Hepatology (June 16-18, 2005, Graz, Austria) (Reference: Dzirlo L, Blaha B, Müller C, Gschwantler M. Morphologische Veränderungen der Dünndarmmukosa aus der Sicht der Kapselendoskopie bei Patienten mit Morbus Whipple. Z Gastroenterol 2005; 43:542).

Endoscopy_UCTN_Code_CCL_1AC_2AH

DOI: $10.1055 / \mathrm{s}-2006-944990$ 


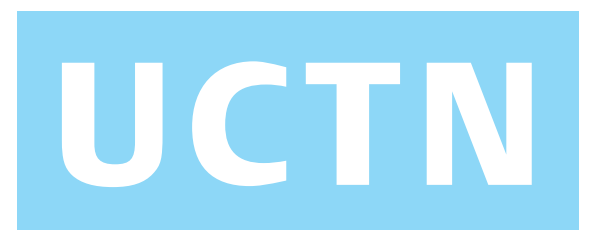

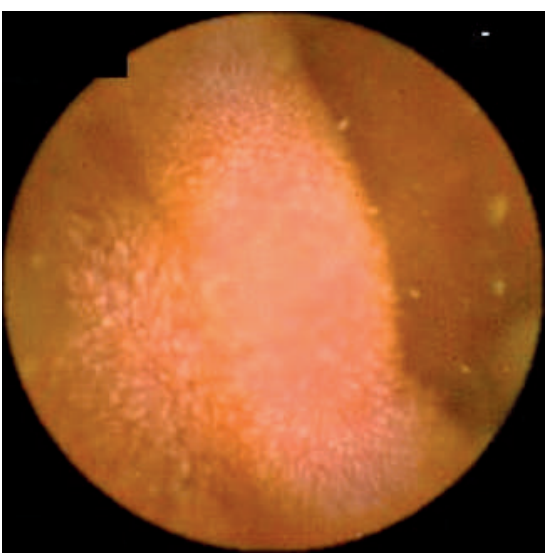

Figure 4 Follow-up capsule endoscopy in patient 1 showed the mucosa in the proximal jejunum to be studded with whitish spots.

\section{Dzirlo', B. Blaha', C. Müller²,}

\section{Hubner ${ }^{3}$, M. Kneussl ${ }^{3}$, K. Huber ${ }^{4}$,}

\section{Gschwantler ${ }^{1}$}

${ }^{1}$ Fourth Department of Internal Medicine, Wilhelminenspital, Vienna, Austria

${ }^{2}$ Department of Internal Medicine IV, Gastroenterology and Hepatology, University of Vienna, Vienna, Austria ${ }^{3}$ Second Department of Internal Medicine, Wilhelminenspital, Vienna, Austria

${ }^{4}$ Third Department of Internal Medicine, Wilhelminenspital, Vienna, Austria.

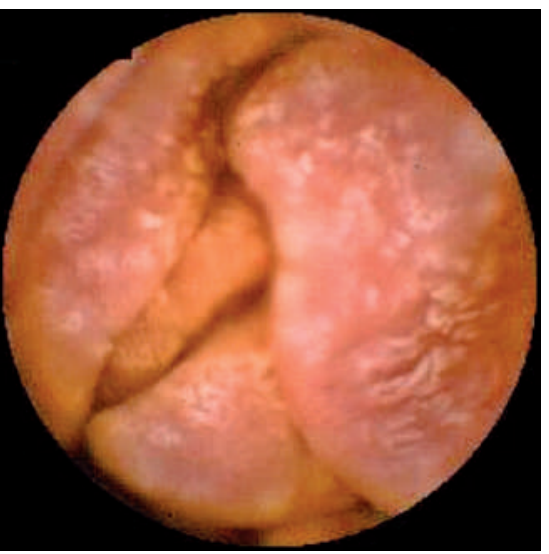

Figure 5 The baseline capsule endoscopy in patient 2 showed a whitish reticular pattern in the proximal jejunum that was suggestive of dilated lymphatic vessels.

\section{References}

${ }^{1}$ Fritscher-Ravens A, Swain CP, von Herbay A. Refractory Whipple's disease with anaemia: first lessons from capsule endoscopy. Endoscopy 2004; 36: 659-662

${ }^{2}$ Gay G, Roche JF, Delvaux M. Capsule endoscopy, transit time, and Whipple's disease. Endoscopy 2005; 37: 272-273

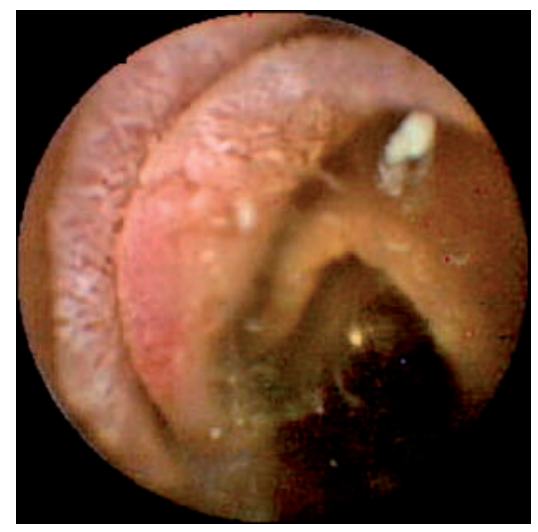

Figure 6 Lymphangiectasias and villous denudation in the proximal jejunum at baseline capsule endoscopy in patient 2.

\section{Corresponding author}

\section{Dzirlo, M. D.}

Fourth Department of Internal Medicine Wilhelminenspital

Montleartstrasse 37

\section{A-1160 Vienna}

Austria

Fax: $\quad+43-1-49150-2409$

Email: larisa.dzirlo@chello.at 